\title{
The limitations of the tendon jerk as a marker of pathological stretch reflex activity in human spasticity
}

\author{
S J Fellows, H F Ross, A F Thilmann
}

\begin{abstract}
The motor disorders associated with human spasticity arise, partly from a pathological increase in the excitability of muscle stretch reflexes. In clinical practice, reflex excitability is commonly assessed by grading the reflex response to a blow delivered to the tendon of a muscle. This is a much simpler response than the complex patterns of activity which may be elicited following muscle stretch caused by active or passive movement. Changes in the biceps brachii tendon jerk response have been followed over the first year after stroke in a group of hemiparetic patients and compared with changes in short and medium latency reflex responses elicited by imposed elbow flexion of initially relaxed spastic muscle and with the development of the late reflex responses which contribute to spastic hypertonia. A progressive increase in tendon jerk responses occurred over the first year following stroke, whereas reflex responses to imposed displacement, in particular the late reflex responses contributing to muscle hypertonia, reached their peak excitability one to three months after stroke, with a subsequent reduction in activity. The tendon jerk reflex therefore provides an incomplete picture of the pathological changes in the reflex responses in spasticity.
\end{abstract}

(F Neurol Neurosurg Psychiatry 1993;56:531-537)

One of the most established tools used by neurologists in routine clinical examination is the use of a hammer to deliver a blow to the tendon of a muscle, activating homonymous muscle spindles which then evoke a synchronised reflex contraction of the muscle, thus providing a measure of reflex excitability. ${ }^{1}$ This activation was held to be mediated over a monosynaptic pathway. Although it has become apparent that oligosynaptic pathways contribute to this response, ${ }^{2}$ and that the afferent volley responsible is not a homogeneous muscle spindle volley, ${ }^{3}$ the tendon jerk reflex does provide an indication of excitability in the simplest reflex pathways. A hammer blow is, however, not a stimulus which the nervous system might expect to encounter in the course of normal behaviour. A more natural stimulus is muscle stretch resulting from imposed displacement of a limb. The response of a muscle to such stretch, particularly during voluntary activation of the muscle, is much more complex than the simple, synchronised burst of the tendon jerk reflex. In addition to short latency components, medium and long-latency reflex components may also be apparent, ${ }^{4}$ mediated over polysynaptic spinal ${ }^{5}$ or transcortical pathways. ${ }^{6}$ Furthermore, in a variety of motor disorders, for example spasticity, even relaxed muscle shows a complex pattern of response to induced stretch. ${ }^{7}$ It is, however, more likely to be the later, more complex responses, for example those contributing to spastic muscle hypertonia, ${ }^{8}$ which cause problems during the performance of movements. This is often apparent during attempted voluntary movement, for example ankle dorsiflexion, where performance of the task may be prevented by stretch reflex activation of the antagonist soleus muscle. ${ }^{9}$ It is therefore interesting to know the extent to which changes in reflex excitability, as accessed by the tendon jerk response, are matched by excitability changes in more functional reflexes. A study of the spastic ankle has revealed that tendon jerk responses on the side ipsilateral to a unilateral ischaemic cerebral lesion (in the area of the middle cerebral artery) provide no indication of a profound depression which can be seen in the stretch reflexes elicited by ankle displacement ${ }^{10}$ on this side, the so-called "good" side. Further, tendon jerk responses on the side contralateral to the lesion show an earlier increase in excitability than the corresponding stretch reflexes elicited by limb displacement.

Accordingly, this study was undertaken to establish the extent to which changes in the Biceps brachii tendon jerk response during the evolution of spasticity following a unilateral cerebral lesion reflect changes in the various components of the response of the Biceps brachii to imposed elbow flexion.

\section{Methods}

The experiments were performed on twenty eight patients who had suffered a unilateral ischaemic lesion in the area of the middle cerebral artery (MCA). In all cases this was the result of the patients' first stroke. A group of twenty normal subjects ( 11 men, 9 women, aged 20-50) with no neurological abnormalities acted as a control. Clinical details of the patients are given in the table. The patients were not receiving anti-spasticity medication during the study, but all received regular physiotherapy, beginning within days of their 
Table Clinical details of the hemiparetic patients

\begin{tabular}{|c|c|c|c|c|c|}
\hline Case & Sex & $\begin{array}{l}\text { Age } \\
\text { (years) }\end{array}$ & Site of lesion & Paresis* & Tonet \\
\hline \multicolumn{6}{|c|}{$<1$ month } \\
\hline 1 & $\mathrm{~m}$ & 64 & rMCA & 2 & 0 \\
\hline 2 & $\mathrm{~m}$ & 62 & $\mathrm{rMCA}$ & 3 & 0 \\
\hline 3 & $\mathrm{~m}$ & 66 & $\mathrm{rMCA}$ & 0 & 0 \\
\hline 4 & $\mathrm{~m}$ & 59 & $\mathrm{rMCA}$ & 0 & 0 \\
\hline 5 & f & 64 & rMCA & 3 & 0 \\
\hline 6 & f & 39 & IMCA & 2 & 1 \\
\hline 7 & $\mathrm{~m}$ & 66 & IMCA & 2 & 1 \\
\hline 8 & $\mathrm{~m}$ & 40 & IMCA & 0 & 0 \\
\hline \multicolumn{6}{|c|}{$1-3$ months } \\
\hline 9 & $\mathrm{~m}$ & 52 & rMCA & 0 & 0 \\
\hline 10 & $\mathrm{f}$ & 70 & IMCA & 2 & 0 \\
\hline 11 & f & 19 & 1MCA & 2 & 1 \\
\hline 12 & f & 49 & rMCA & 0 & 3 \\
\hline 13 & f & 75 & rMCA & 0 & 4 \\
\hline 14 & $\mathrm{~m}$ & 64 & rMCA & 1 & 2 \\
\hline 15 & $\mathrm{~m}$ & 58 & $1 \mathrm{MCA}$ & 4 & 2 \\
\hline 16 & $\mathrm{~m}$ & 61 & $1 \mathrm{MCA}$ & 1 & 3 \\
\hline 17 & $\mathrm{~m}$ & 78 & $\mathrm{rMCA}$ & 0 & 4 \\
\hline 18 & $\mathrm{f}$ & 51 & $\mathrm{rMCA}$ & 4 & 0 \\
\hline \multirow{2}{*}{\multicolumn{6}{|c|}{$>1$ year }} \\
\hline & & & & & \\
\hline 20 & $\mathrm{~m}$ & 56 & 1MCA & 0 & 3 \\
\hline 21 & $\mathrm{~m}$ & 33 & rMCA & 4 & 2 \\
\hline 22 & $\mathrm{~m}$ & 47 & IMCA & 4 & 4 \\
\hline 23 & $\mathrm{f}$ & 48 & $1 \mathrm{MCA}$ & 4 & 4 \\
\hline 24 & f & 50 & IMCA & 0 & 4 \\
\hline 25 & f & 51 & $1 \mathrm{MCA}$ & 3 & 4 \\
\hline 26 & f & 40 & rMCA & 0 & 1 \\
\hline 27 & $\mathrm{~m}$ & 47 & rMCA & 4 & 3 \\
\hline 28 & $\mathrm{~m}$ & 38 & rMCA & 4 & 3 \\
\hline
\end{tabular}

IMCA = left middle cerebral artery, rMCA = right middle cerebral artery. Localisation of the lesion was established in all cases by CT and, in most cases, by angiography and/or magnectic

resonance imaging.
$\star$ Paresis of the affected biceps brachii, expressed according to the MRC scale: $0=$ no voluntary power; 1 = visible contraction without affect; $2=$ movement without the influence of gravity; $3=$ movement against gravity; $4=$ movement against resistance; $5=$ normal power.

$3=$ movement against gravity; $4=$ movement against resistance; $5=$ normal power.
Tone of the spastic arm during passive extension, expressed on the Ashworth ${ }^{11}$ scale: Tone of the spastic arm during passive extension, expressed on the Ashworth" scale:
$0=$ normal tone; 1 = slight increase in tone, giving a "catch" when the limb is moved; $0=$ normal tone; $1=$ slight increase in tone, giving a "catch" when the limb is moved;
$2=$ more marked increase in tone, but limb still easily moved; $3=$ considerable increase in $2=$ more marked increase in tone, but limb still easily m
tone-passive movement difficult; $4=$ limb rigid in extension

stroke. The patients were grouped according to the time elapsed since stroke, the periods studied being less than one month, one to three months and more than one year. The patients and normal subjects gave their informed consent to all procedures, which had previously been approved by the local ethical committee.

The subjects were seated in a stable chair, with the arm laterally abducted and the forearm and hand enclosed in a plastic mould, restrained by a series of straps. The height of the chair was adjusted so that the shoulder was level with, or slightly above the level of the arm. The mould was in turn fixed to an aluminium support which was mounted on a bearing adjusted to be in alignment with the axis of the elbow joint. The mould was joined at the wrist, via a rigid rod, to an electric motor (Brown Boveri, MC24P, 3000W, Germany) operating under a four-quadrant control system (Seidel, series 5000, Germany). This motor was capable of applying elbow extension at a variety of velocities, the displacement arising from a given set of movement parameters being identical for all subjects, irrespective of arm inertia or any reflex forces developed. The parameters were supplied by a laboratory computer (DEC, PDP11/73, USA). Displacement amplitude was $30^{\circ}\left(75^{\circ}\right.$ flexion to $\left.105^{\circ}\right)$, applied at 300 , $240,175,115$ or $80 \%$ s. Force was measured by a piezo-electric transducer (Kistler 9311A, Switzerland) placed in series with the rod connecting the motor and the cast. Position was monitored using a light-sensitive detector (United Detector Technology, LSC5D, USA) which followed a light source rigidly attached to the connecting rod. Electromyograms (EMG) were recorded from the biceps brachii $(\mathrm{Bb})$ and triceps brachii $(\mathrm{Tb})$ muscles using custom-built surface electrodes which contained a small pre-amplifier designed to eliminate movement artifacts. The signals so obtained were then amplified and filtered (Bandwidth $20 \mathrm{~Hz}-1 \mathrm{KHz}$ ).

Ten displacements were applied at each displacement velocity. The subjects were asked to relax their arm muscles and were instructed not to resist the imposed displacement. That they complied with these instructions was established by monitoring the EMG signals on an oscilloscope. Any trial in which EMG was present at the onset of displacement was discarded. Displacement was initiated by the computer, which sampled, via its $\mathrm{ADC}$ boards, position, force and $\mathrm{Bb}$ and $\mathrm{Tb}$ EMG signals with a rate of $1 \mathrm{KHz}$. These signals, with the motor control pulse, were also recorded on tape (Racal Store 7D, UK). The computer rectified the EMG signals and constructed an average from the ten displacements applied at each velocity. All data were written to disc for subsequent analysis.

For the patients, the responses were evaluated in terms of integrated EMG activity (in $\mu \mathrm{V} . \mathrm{s})$ in three segments, defined according to their timing relative to the "normal range" (61-107ms: that is the average onset and end of the response to $300 \%$ displacement in normal subjects: $\operatorname{see}^{78}$ for a full definition), namely "early" activity, appearing before this time; "normal range" activity, that falling within this interval; and "late" activity, occuring after this interval, up to the end of the displacement. For further clarity, fig 1 shows the

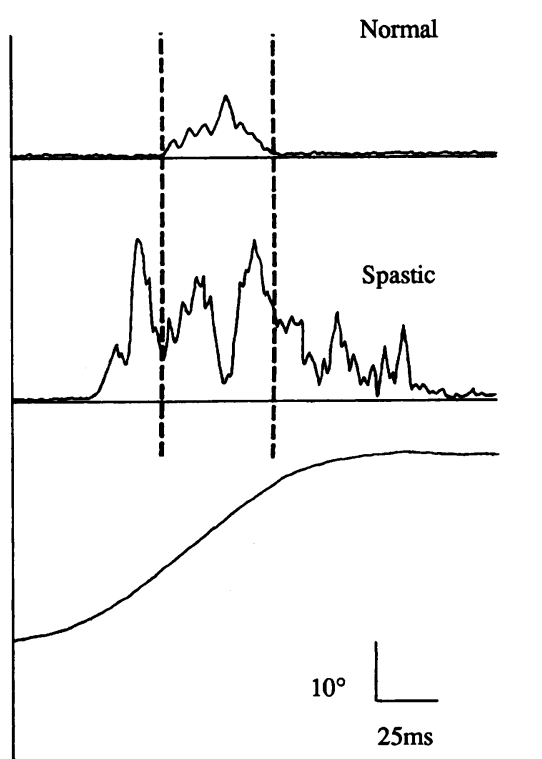

$50 \mu \mathrm{V}$

Figure 1 The upper and middle traces show the rectified and averaged EMG responses of the relaxed $B b$ of $a$ representative normal subject and patient (23 in table 1), respectively, obtained from ten displacements at $300 \%$. The dotted lines indicate the timing of the "normal range" activity (61-107ms). The lower curve shows the position curve for the applied displacement. 
Figure 2 The group means of the area of the $B b$ tendon jerk reflex $E M G$ (in $\mu V s$, with standard error bar) for the normal subjects and for the three groups of patients.

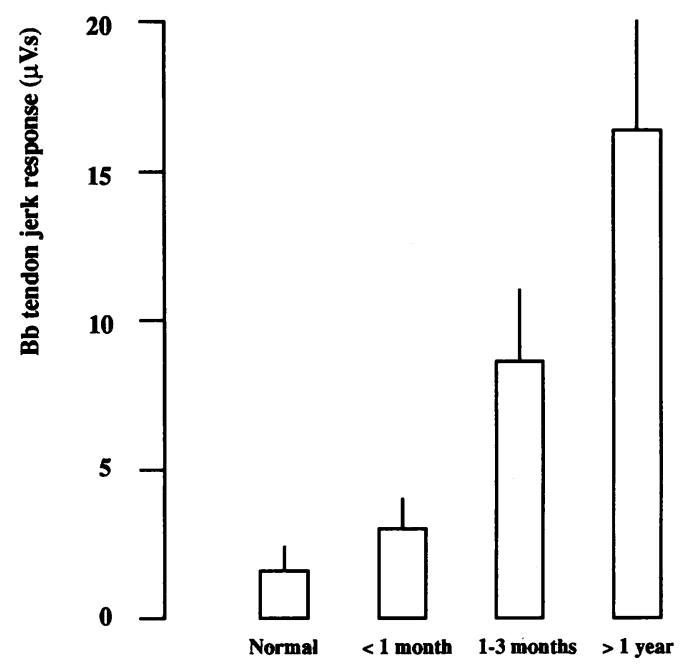

rectified EMG curves obtained following displacement at $300 \%$ sor a representative normal subject and a patient. The dotted lines indicate the timing of the "normal range". In the case of the "late" activity, the integral values so obtained were then divided by the duration of the activity to yield a mean level of EMG activity (in $\mu \mathrm{V}$ ).

a)

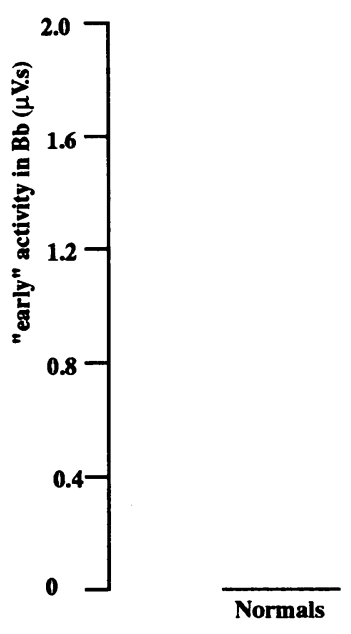

b)
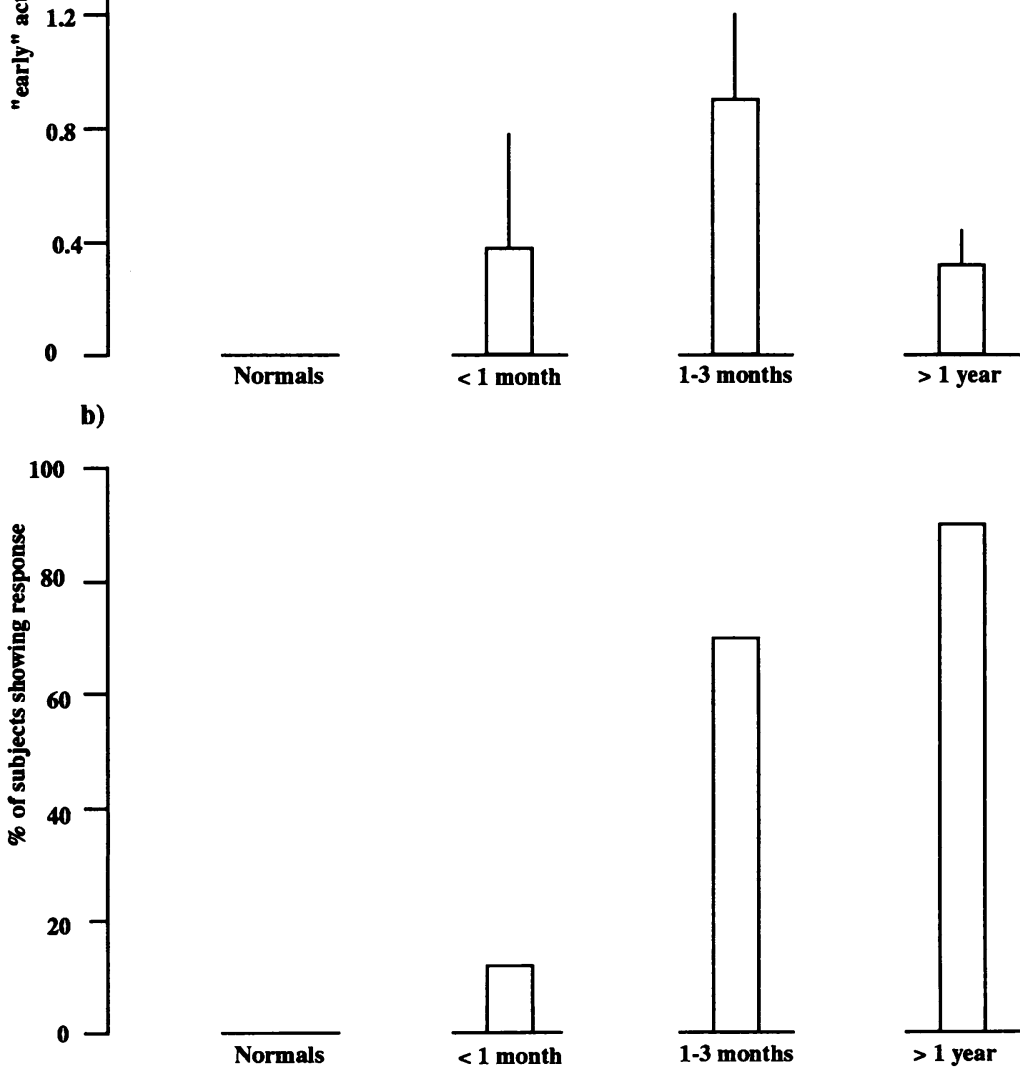

Figure 3 A) The group means (with standard error bar) of the area of the "early" activity seen in the $B b$ EMG following an imposed extension of the initially relaxed arm of $30^{\circ}$ at $300 \mathrm{deg} / \mathrm{s}$. B) The percentage of subjects in each group showing Bb EMG activity in this period.
$\mathrm{Bb}$ tendon jerk responses were obtained with the subject still positioned in the mould, which was fixed in the middle of the displacement range (that is, $90^{\circ}$ ). Responses were elicited by an experienced neurologist, using manual percussion of the $\mathrm{Bb}$ tendon. Preliminary testing had established that such a procedure had a high degree of reproducibility. The blow was delivered with a reflex hammer in whose head was a small microswitch which closed on contact and initiated computer sampling of the EMG responses. Five responses were obtained. The EMG record from each was rectified and then averaged and the area of the integrated EMG record (in $\mu \mathrm{V} . \mathrm{s}$ ), its latency and duration (in $\mathrm{ms}$ ) were measured.

Statistical analysis was performed using variance analysis of the group data or the Spearman Rank correlation test (Statview II, Abacus Concepts, USA).

\section{Results}

TENDON JERK RESPONSES

A tendon jerk response could be evoked in all of the patients and in all but one of the normal subjects. The group averages and standard errors are displayed in fig 2 .

In the first month after stroke, the amplitude of the tendon jerk response increased above normal values, although this increase did not yet reach statistical significance. The reason for this lack of significance is to be found in the wide overlap of the respective ranges of values: the largest tendon jerk responses obtained from the normal subjects reached similar values to the maximum responses of the patients tested $<1$ month after stroke. If the percentiles of the two groups were compared, however, it was apparent that the patient responses were larger in every percentile up to the $80 \%$ level, after which the percentiles were very similar. Although the tendon jerk responses of the patients tested $<1$ month after stroke did not fall outside the range of normal values, their responses lay at the upper end of this range. Between 1-3 months after stroke, the tendon jerk reflex was markedly exaggerated $(p<$ $0.025)$. By the end of the first year after stroke this increase had progressed still further $(p<0.001)$ reaching values which, on average, were eight times greater than those seen in normal subjects. At no time were significant changes observed in the latency [Mean (SD) values: normals 12(4) $\mathrm{ms}$; <1 month 13(4); 1-3 months $13(4)$; $>1$ year $11(1)$ ] or the duration [Mean (SD) values; normal subjects $20(6)$; <1 month $10(4)$; 1-3 months 22(4)] of the tendon jerk response.

\section{“EARLY" ACTIVITY}

As "early" activity (that occuring before $61 \mathrm{~ms}$ ) was not observed in the normal subjects, its amplitude in the patients cannot be compared to normal values. Comparison between the patient groups, however, can be performed, and the resulting averages ( \pm SEM) obtained at the fastest rate of dis- 
a)

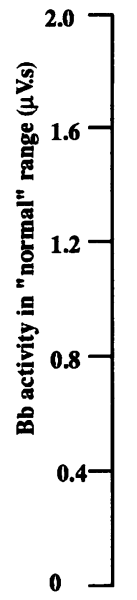

b)

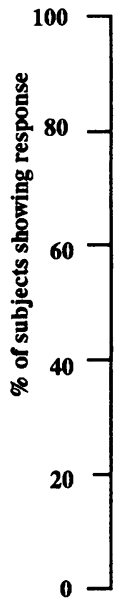

$\left.\begin{array}{l}100 \\ 80-5 \\ 60 \\ 40 \\ 20 \\ 0\end{array}\right]$
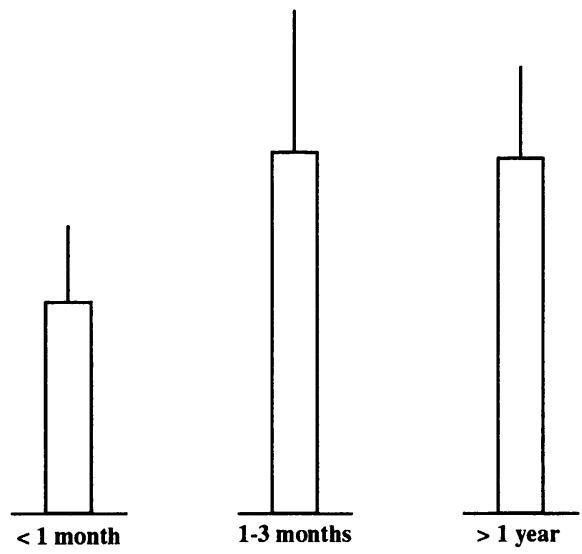
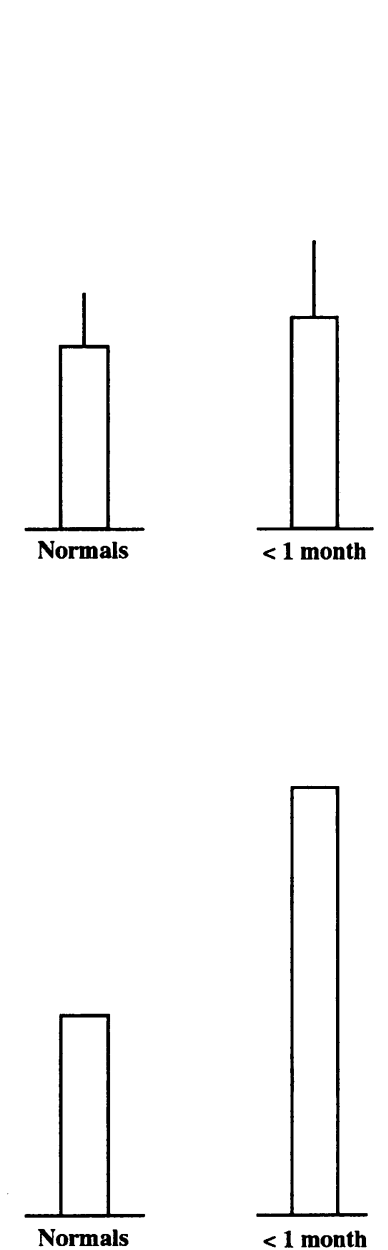
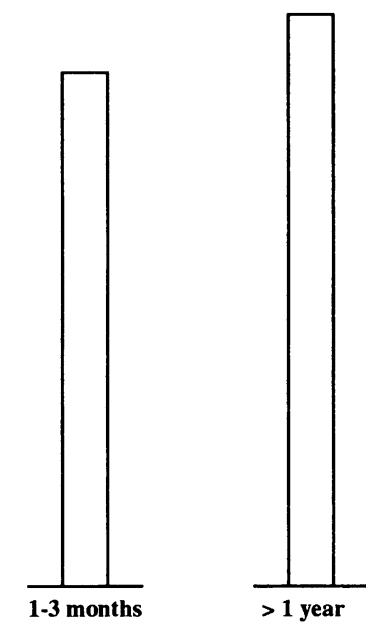

Figure 4 A) The group means (with standard error bar) of the area of the Bb EMG activity seen in the "normal range" following an imposed extension of the initially relaxed arm of $30^{\circ}$ at $300 \mathrm{deg} / \mathrm{s}$. B) The percentage of subjects in each group showing Bb EMG activity in this period.
ACTIVITY IN THE "NORMAL RANGE"

Displacement at $300 \%$ s elicited a reflex EMG in $35 \%$ of the normal subjects. For the reasons given above, subjects not showing a response were excluded from the group average. The group mean of the normals, along with those of the three patient groups are shown in fig 4a.

No difference existed between the "normal activity" in patients in the first month after stroke and the amplitude of the reflex EMG of the normal subjects. After the first month, the level of response increased, but this did not reach significant levels. If the percentage of subjects showing a response is calculated, however, it is apparent (fig 4b) that an increase occurs within weeks of a stroke, with a subsequent progression until, by the end of the first year after stroke, all patients demonstrated activity in the "normal range". Thus the alteration in this response in spastic subjects consists not of a significant increase in the level of response, but rather in the abnormally high occurrence of this response, which becomes ever more marked over the course of the first year after stroke.

Associated with this increase in the number of patients showing a response is a steady decrease in the lowest displacement velocity at which a response is first evident.

Figure 5 shows the distributions of the reflex threshold of activity in the "normal range" for the normal subjects and for the three patient groups. It may be seen that while no normal subject showed a reflex response with displacement velocities lower than $175 \%$, by the end of the first year after stroke a large percentage $(78 \%)$ responded to displacement at $115 \%$, and a minority still showed activity with stretch velocities as low as $80 \%$ s.

\section{“LATE" ACTIVITY}

As would be expected from a previous study, ${ }^{8}$ all patients in the groups tested 1-3 months or $>1$ year after stroke who were clinically assessed to have raised muscle tone in the $\mathrm{Bb}$ responded to imposed stretch with "late" reflex EMG activity. This began immediately after the "normal range" (107ms) and terminated at or around the end of the displacement. The mean level of this EMG activity showed a highly significant positive linear correlation with displacement velocity. As has previously been shown, ${ }^{8}$ the slope of the regression plot between mean Bb EMG and velocity represents the gain of the reflex mechanism underlying this activity. Additionally, the mean level of the "late" EMG activity showed a significant linear positive correlation to the passive muscle hypertonia clinically assessed in these patients on the Ashworth Scale ${ }^{11}(1-3$ months $r=0 \cdot 88$, $\mathrm{p}=0.008 ;>1$ year $\mathrm{r}=0.7, \mathrm{p}=0.048)$. In the patient group tested in the first month after stroke, although hypertonia was positively scored on the Ashworth scale in only two subjects, several others also showed some "late" Bb EMG activity. In these cases, however, in common with the two subjects in 


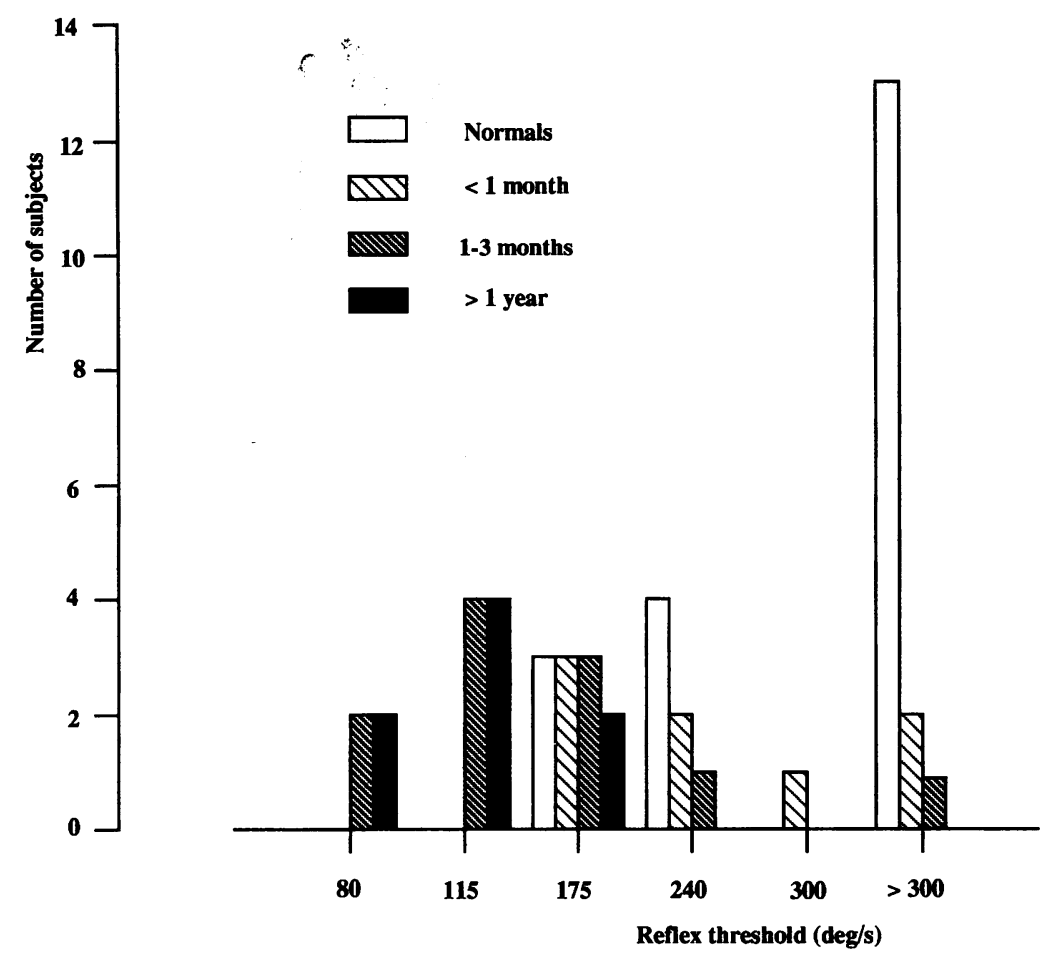

Figure 5 The reflex thresholds of the normal subjects and the three patient groups. Threshold is defined as the lowest of the five displacement velocities applied which elicited a response in a given subject. $>300$ indicates that no reflex response was obtained with the highest velocity used. group showing late $\mathrm{Bb}$ EMG $(\mathrm{n}=5)$ were included in the statistical analysis.

Figure 6 shows the mean level of Bb EMG ( \pm SEM) during the "late" response elicited during displacement at $300 \%$ for the three groups. It is apparent that in the first month of spasticity, only a very low level of Bb EMG activity was evoke 1 . Between the first and third month after stroke, however, the level of "late" Bb EMG increased considerably $(p<0.01)$. Such an increase is in line with the behaviour of the tendon jerk reflex at this time, but whereas the tendon jerk subsequently showed a further increase in patients a year or more after stroke, it may be seen in fig 6 that the "late" Bb EMG obtained in these patients was considerably reduced from the level of activity seen in the patients 1-3 months after stroke $(p<0.01)$. Thus a disparity exists between the behaviour of the tendon jerk reflex and the late reflex EMG activity which contributes to one of the major motor disturbances associated with spasticity, namely muscle hypertonia.

\section{INTRA-INDIVIDUAL DIFFERENCES}

The previous sections have established that, for group mean values, clear differences exist in the behaviour of tendon jerk reflexes and a variety of reflexes elicited by limb displacement. It is valid to ask therefore if the tendon jerk of individual patients reflected the level of response of the reflex responses to imposed displacement. To answer this question a comparison was made between the size of the different reflex components in the various groups. It was found that for the patients tested less than one month or more than one year after stroke, the magnitude of the tendon jerk response showed no significant correlation with the magnitude of any of the reflex components obtained by limb displacement (no $p$ values smaller than 0.383 ). For the group tested between 1-3 months after stroke, the magnitude of the tendon jerk response was positively correlated with the "early" activity $(p=0.016)$ and the activity elicited in the "normal range" $(p=0.001)$. No significant correlation, however, was apparent with the mean level of the "late" reflex activity ( $p=$ 0.127).

\section{Discussion}

The results of this study agree with an earlier finding obtained at the hemiparetic ankle, ${ }^{10}$ that the changes in the tendon jerk response during the development of spasticity do not inevitably reflect alterations in more naturally elicited stretch reflex responses, such as those resulting from limb displacement. This finding has important implications not only for clinical assessment of spasticity, but also for studies of the pathophysiological mechanisms underlying spasticity and for studies intended to evaluate the efficacy of therapeutic intervention, whether pharmacological or physical.

The finding that the tendon jerk response of the patient group tested $<1$ month after stroke was not significantly elevated over nor- 
mal values might, at first sight, be considered surprising from a clinical viewpoint: it is generally accepted that exaggerated tendon jerks are first apparent within days, rather than weeks of the causative lesion. Furthermore, experimental studies in the rat and cat have demonstrated that monosynaptic reflexes are significantly increased almost immediately following a spinal cord lesion. ${ }^{12}{ }^{13}$ It must be remembered, however, that clinical assessment of elevated tendon jerk reflexes is performed as a comparison of the two sides of a patient. A recent study has emphasised, however, that this cannot be considered to be the same as a comparison against normal values, on the grounds that the $\mathrm{Bb}$ tendon jerk response ipsilateral to a strictly unilateral cerebral lesion is significantly reduced from normal values. ${ }^{7}$ Thus the comparison of the $\mathrm{Bb}$ tendon jerk responses on both sides of a patient with a unilateral cerebral lesion may lead to an exaggerated impression of the increase on the side contralateral to the lesion, relative to the actual increase over normal control values. In addition, serial studies of H-reflex changes following spinal cord injury in humans have revealed that significant increase in a measure of $\mathrm{H}$-reflex excitability, the $\mathrm{H} / \mathrm{M}$ ratio, are first apparent 4-6 months after injury. ${ }^{14}$ The same group have also shown in experiments on the cat ${ }^{15}$ that the increase in monosynaptic reflex excitability following spinal cord transection is biphasic, with an early increase 1-4 days after transection, followed by a more significant rise between 2 and 4 weeks later. Although the situation following spinal transection may not be fully comparable with that pertaining after stroke, this biphasic pattern of increase may be reflected in the results of the present study. The earliest increase may be reflected in the observed tendency for the $\mathrm{Bb}$ tendon jerk response amplitudes of patients tested $<1$ month after stroke to cluster at the upper end of the normal range. The second, more marked phase of increase may then be apparent as the significant increase of the tendon jerk response seen in the patients tested 1-3 months after stroke.

The progressive increase in the magnitude of the tendon jerk response over the course of the first year after stroke does not reflect the more complicated changes in the "late" EMG responses contributing to spastic muscle hypertonia, which decreases after reaching a maximum 1-3 months after stroke, nor the relatively unaltered state of shorter latency reflexes elicited by limb displacement. The steady increase is, however, mirrored by the number of patients in whom shorter latency responses to limb displacement were to be seen. At first sight the connection between an increase in tendon jerk response magnitude and the frequency of occurrence of responses which do not show parallel changes in magnitude may not seem obvious. If, however, the likely pathways of the various responses are considered, a possible explanation becomes apparent. While the tendon jerk response is mediated over monosynaptic and oligosynap- tic connections, ${ }^{2}$ the latency or the reflex activity observed in the "normal range" would indicate that there exists a significant interneuronal contribution to this response. Although, given its short onset latency (as early as $25 \mathrm{~ms}$ after the onset of displacement), the "early" activity is likely to be mediated at least in part over monosynaptic and oligosynaptic connections, the absence of a progressive increase in the magnitude of this activity would indicate that other connections, at a more complex level than that subserving the tendon jerk response and subject to a wider range of influences, are also involved in this response. It is possible therefore that the increase in the tendon jerk response reflects excitability changes at a very basic level of the motor system. Changes in receptor excitability, pre-synaptic inhibition of afferent transmission and motor neuron excitability have all been considered as possible mechanisms of spastic hyperreflexia. Clear evidence has, however, only been found for the second of these suggestions: the inhibition of $\mathrm{H}$-reflex responses by tendon vibration, which is thought to be mediated by a pre-synaptic mechanism, has been shown to be greatly reduced in spastic patients. ${ }^{1617}$ Microneurographic recordings of muscle spindle afferents in spastic humans have failed, as yet, to demonstrate any evidence of receptor hyperexcitability in the relaxed state. ${ }^{18}$ Such a receptor hyperexcitability, for example in the muscle spindle endings would, however, be compatible with the observed increases in the amplitude of monosynaptic reflex responses and could also explain the reduction in threshold and increase in the late reflex components mediated over pathways with greater interneuronal involvement. Under this schema, an increase in receptor sensitivity would lead to the drop in the threshold of these later components and to an increase in the number of patients in whom they were observed. The different timecourse of alterations in the magnitude of the later components would then reflect additional influences operating on the more complex parts of their pathways (that is, the interneurons), which are not apparent at a mono- or oligo-synaptic level. Until evidence for increased receptor sensitivity is provided, however, such an explanation must remain speculative.

An obvious question which arises from this disparity between the behaviour of the tendon jerk reflex and more naturally elicited reflex responses is, what relevance does it have for the patient and the clinician? Clearly, in clinical practice a diagnosis of spasticity is unlikely to be made on the basis of exaggerated tendon jerk reflexes alone, but in many studies attempting to evaluate the efficacy of physical or pharmaceutical intervention, evaluation of changes in reflex behaviour are often made on just such a basis, from either tendon jerk or H-reflex studies. The results of our study indicate that investigations restricting themselves to these simple peripheral pathways will either fail to reveal, or run the risk of mis- 
interpreting, the complex changes occurring in more physiological reflex responses, which cause the patient far more problems than alterations in a non-physiological parameter such as the tendon jerk reflex. ${ }^{9}$ Similarly, although it has been shown that short-latency ("monosynaptic") reflexes generated in spastic patients during gait contribute little or no resisting force to an imposed displacement, ${ }^{19}$ it would be wrong, on the basis of the behaviour of monosynaptic reflex responses alone, to state that hyperreflexia plays no part in the motor disability of the spastic subject. ${ }^{20}$ It is apparent that future studies must be concentrated on the more complex, movement evoked reflex responses of the motor system if a correct picture of the pathophysiology of spasticity is to be successfully achieved, and if likely modes of therapy are to be assessed and the success of such intervention correctly evaluated.

This work was supported by the Deutsche Forschungsgemeinschaft. We thank Elisabeth Garms for her Noth and Drs Dömges, Schwarz and Töpper for their helpful comments on the manuscript.

1 Lansaka DJ. The history of reflex hammers. Neurology 1989;39:1542-9.

2 Burke D, Gandevia SC, McKeon B. Monosynaptic and oligosynaptic contributions to human ankle jerk and Holigosynaptic contributions to human
reflex. F Neurophysiol 1984;52:435-48.

3 Burke D, Gandevia SC, McKeon B. The afferent volleys responsible for spinal proprioceptive reflexes in man. $f$ Physiol 1983;339:535-52.

4 Lee RG, Tatton WG. Motor responses to sudden limb displacements in primates with specific CNS lesions and in human patients with motor system disorders. Can $\mathcal{F}$ Neurol Sci 1975;2:285-93.
5 Hultborn H, Wigström H. Motor response with long latency and maintained duration evoked by activity in Ia afferents. In: Desmedt JE, ed. Super and supraspinal mechanisms of voluntary motor control and locomotion. Progress in clinical Neurophysiology 8. Basel: Karger, Progress in clini

6 Marsden CD, Merton PA, Morton HB. Stretch reflex and servo action in a variety of human muscles. $\mathcal{F}$ Physiol servo action in a

7 Thilmann AF, Fellows SJ, Garms E. Pathological stretch reflexes on the "good" side of hemiparetic patients. $f$ Neurol Neurosurg Psychiatry 1990;53:208-14.

8 Thilmann AF, Fellows SJ, Garms E. The mechanism of spastic muscle hypertonus: variation in reflex gain over the time course of spasticity. Brain 1991;114:233-44.

9 Corcos DM, Gottlieb GL, Penn RD, Myklebust B, Agarwal GC. Movement deficits caused by hyperexcitable stretch reflexes in spastic humans. Brain 1986;109:1043-58.

10 Thilmann AF, Fellows SI. The time-course of bilateral changes in the reflex excitability of relaxed triceps surae changes in the reflex excitability of relaxed triceps surae muscle in human

11 Ashworth B. Preliminary trial of Carisoprodol in multiple sclerosis. Practitioner 1964;192:540-2.

12 Malmsten J. Time course of segmental reflex changes after chronic spinal cord hemisection in the rat. Acta Physio Scand 1983;119:435-43.

13 Chambers WW, Lui C-N, McCouch GP, D'Aquili E Descending tracts and spinal shock in the cat. Brain 1966;89:377-90.

14 Little LW, Harlar EM. H-reflex changes following spinal cord injury. Arch Phys Med Rehabil 1985;66:19-22.

15 Little LW. Serial recording of reflexes after feline spinal cord transection. Exp Neurol 1986;93:510-21.

16 Ashby P, Verrier M, Carleton S, Somerville J. Vibratory inhibition of the monosynaptic reflex and presynaptic inhibition in man. In: Feldman RG, Young RR, Koella Whibition in man. In: Feldman RG, Young RR, Koella Wear eds. Spasticity: disordered mok Medical, 1980:335-44

17 Iles JF, Roberts RC. Presynaptic inhibition of monosynaptic reflexes in the lower limbs of subjects with upper motoneuron disease $f$ Neurol Neurosurg Psychiatry 1986;49:937-44.

18 Hagbarth K-E, Wallin G, Löfstedt L. Muscle spindle responses to stretch in normal and spastic subjects. Scand F rehab Med 1979;5:156-9.

19 Berger W, Horstmann GA, Dietz V. Tension development and muscle activation in the leg during gait in spastic hemiparesis: the independence of muscle hypertonia hemiparesis: the independence of muscle hypertonia and exaggerated stretch reflex

20 Dietz V. Spastik: Therapie der gesteigerten Reflexe oder der Bewegungsstörung? Nervenartz 1990;61:581-6. 